Oerlemans, S., Cranenburgh, O. van, Herremans, P.J., Spreeuwenberg, P., Dulmen, S. van. Intervening on cognitions and behavior in irritable bowel syndrome: a feasibility trial using PDAs. Journal of Psychosomatic Research: 2011, 70(3), 267-277

\begin{tabular}{|l|l|}
\hline Postprint Version & 1.0 \\
\hline Journal website & $\underline{\text { http://dx.doi.org./10.1016/j.jpsychores.2010.09.018 }}$ \\
\hline Pubmed link & $\underline{\text { http://www.ncbi.nlm.nih.gov/pubmed/21334498 }}$ \\
\hline DOI & 10.1016/j.jpsychores.2010.09.018 \\
\hline
\end{tabular}

This is a NIVEL certified Post Print, more info at http://www.nivel.eu

\title{
Intervening on cognitions and behavior in irritable bowel syndrome: A feasibility trial using PDAs th
}

\author{
Simone Oerlemans ${ }^{\mathrm{A}}$, OdA VAN CRANENBURGH ${ }^{\mathrm{A}}$, Pieter-JAN HERREMANS ${ }^{\mathrm{A}}$, PETER SPREEUWENBerG ${ }^{\mathrm{A}}$ \\ AND SANDRA VAN DULMEN *, ${ }^{\text {A }}$ \\ ${ }^{a}$ NIVEL (Netherlands Institute for Health Services Research), Utrecht, The Netherlands
}

\begin{abstract}
Objective: Irritable bowel syndrome (IBS) is a common, chronic, and often disabling disorder. Cognitive-behavioral therapy (CBT) has been shown to be effective in alleviating IBS symptoms. The aim of this study was to establish the feasibility and the efficacy of a cognitive-behavioral e-intervention using personal digital assistants (PDAs) on the selfmanagement of IBS patients.

Methods: A feasibility trial was conducted with 38 control group patients receiving standard care and 37 intervention group patients receiving standard care supplemented with a 4-week CBT intervention on PDAs. All patients fulfilled the diagnostic Rome III criteria for IBS. At baseline, 4-week, and 3-month follow-up, patients' abdominal pain, dysfunctional cognitions, IBS quality of life, and pain catastrophizing thoughts were assessed using written questionnaires.

Results: Between-group comparisons between baseline and 4 weeks showed more overall quality of life improvement, more improvement in catastrophizing thoughts, and more pain improvement in the intervention group. Only improvement in catastrophizing thoughts persisted in the long-term. No significant differences between groups were found for dysfunctional cognitions. As all 37 intervention group patients completed the intervention and completed diaries three times a day for 4 weeks, the e-health intervention seems feasible.

Conclusions: A cognitive-behavioral intervention on pocket-type computers appears feasible and efficacious for improving IBS-related complaints and cognitions in the short-term. The intervention group improved on several aspects, but most on catastrophizing thoughts, and these improvements even persisted after 3 months. Future studies should focus on unravelling the effective components of this innovative e-health intervention.
\end{abstract}

\section{INTRODUCTION}

Irritable bowel syndrome (IBS) is a functional bowel disorder in which abdominal pain or discomfort is associated with defecation or a change in bowel habit, and with features of disordered defecation [1]. Abdominal pain and bloating are considered as the most annoying symptoms [2]. IBS is one of the most common clinical problems encountered by general practitioners (GPs) and gastroenterologists. In the 
Oerlemans, S., Cranenburgh, O. van, Herremans, P.J., Spreeuwenberg, P., Dulmen, S. van. Intervening on cognitions and behavior in irritable bowel syndrome: a feasibility trial using PDAs. Journal of Psychosomatic Research: 2011, 70(3), 267-277

general population, the prevalence rate of IBS is $10-25 \%$ and women are twice as often affected as men [3] and [4]. One third of these persons seek medical counsel for IBS [5].

So far, IBS does not appear to be associated with any biochemical, structural, or serologic abnormalities that define its presence [6]. Because reliable biological markers are not available either, the diagnosis is based on symptomatic criteria such as the Rome III criteria [7]. Although some medical treatments for IBS, such as smooth-muscle relaxants, have shown relief [8] and [9], no single medication has proven to be universally effective [10].

Enhancing appropriate self-management appears to be valuable [11], [12] and [13] in terms of a reduction in the use of health care services [14] and an improvement of patients' symptoms and quality of life [15]. As the burden of symptoms seems to be associated with symptom-related cognitions and behaviors, an essential part of effective self-management is the restructuring of these dysfunctional cognitions and behaviors, like catastrophizing thoughts and avoidance behavior (in terms of avoiding activities or avoiding specific nutrition), through cognitive-behavioral therapy (CBT). CBT is considered to be more effective than usual care in relieving IBS symptoms [16].

CBT teaches patients how events, thoughts, emotions, actions, and physiological responses are related. It is oriented towards change and development of new skills and strategies for coping with problems [17]. CBT has shown to enhance self-management in terms of successful cognitive and behavioral coping and of pain in a number of IBS patients [18], [19], [20] and [21]. However, the effects of CBT on social functioning seem less convincing [20]. An explanation for this might be that most CBT interventions take place on a weekly basis or even less. This means that in CBT, patients usually receive retrospective and nonsituational feedback on their cognitions and behaviors. Providing immediate, situational feedback at the moment dysfunctional cognitions and behaviors occur may increase the patient's self-management skills and help alleviate their abdominal complaints.

A valid and reliable way for assessing complaints and complaint-related cognitions and behaviors as they occur is by maintaining daily symptom diaries. Daily symptom diaries assess complaints and complaintrelated behavior at the moment they occur, thereby limiting retrospection bias. Thereby, ecologic validity is enhanced as assessment takes place in the natural environment. As complaints and complaint-related behavior are assessed repeatedly, diaries are especially valuable in capturing fluctuating psychological and physical states. Diaries have been used with patients with different complaints, such as migraine [22], low back pain [23], and IBS [19], [24] and [25]. However, so far, most diaries are developed as a paper-andpencil method and as these data are not immediately available for health care providers, tailored and situational feedback cannot be provided directly after completing the diaries. Electronic diaries on personal digital assistants (PDAs; i.e., pocket-type computers) have many advantages: PDAs guarantee reliable storage of data, immediate transfer of data to researcher or counselor, and a transmission of feedback tailored to the data being sent [26] and [27]. Besides, the compliance with completing diaries on PDAs is generally high [28].

The purpose of the current feasibility trial was to establish the efficacy of a cognitive-behavioral intervention using PDAs on the self-management of IBS patients. The feedback intervention focuses on opposing well-known features of inadequate self-management in IBS: dysfunctional cognitions, catastrophizing thoughts, and avoidance behavior. It is expected that this e-health intervention improves dysfunctional cognitions, IBS quality of life, and complaint severity more than standard care (e.g., education, diet, reassurance) alone.

\section{METHODS}

\section{Patients}

Between March 2007 and July 2008, IBS patients in The Netherlands were recruited for this study by GPs and through advertisements by the Dutch IBS patient association. Patients were eligible for this study if they had been suffering from IBS according to the International Classification of Primary Care (ICPC: D93) or Rome III criteria [7] for at least 3 months, were sufficiently acquainted with the Dutch language, had no visual handicap, and were between 18 and 65 years old. Patients were excluded if they had no IBS, had another serious condition (like cancer, cardiovascular diseases, other GI diseases, or psychological disorders), or were currently receiving psychological treatment of any kind, as this could obscure or distort the effects of the proposed CBT intervention. 
Oerlemans, S., Cranenburgh, O. van, Herremans, P.J., Spreeuwenberg, P., Dulmen, S. van. Intervening on cognitions and behavior in irritable bowel syndrome: a feasibility trial using PDAs. Journal of Psychosomatic Research: 2011, 70(3), 267-277

A total of 116 patients were willing to participate in the study, of whom 17 were excluded because they did not meet the inclusion criteria (14.7\%). Of the remaining eligible patients, a further 11 were excluded due to nonresponse before inclusion (11.1\%), another six had lost their interest before inclusion $(6.1 \%)$, five underestimated the required effort (5.1\%), and one found the PDA too difficult to handle during the personal instruction meeting (1.0\%), leaving a total group of 76 patients from whom we got informed consent (see Fig. 1). The study protocol was approved by the medical ethics committee and registered in the Netherlands Trial Register with number ISRCTN09185998.

\section{Design}

The present study was designed and conducted as an open-label RCT. Block randomization was carried out in 76 patients. Within this type of randomization balance is enforced, so that there are similar number of patients assigned to the experimental and control group. MS Excel was used to generate a randomization list allocating patients either to the intervention or to the control group in order of informed consent date. All control group patients and intervention group patients completed similar questionnaires and sent them in using a reply envelope only marked with their (blinded) ID number. The intervention followed an $\mathrm{AB}$ design: a 1-week diary-monitoring period without feedback to get used to the PDA and diaries (A) and a 3week diary-monitoring period with feedback (B).

\section{Sample size}

Based on previous observations, an $\alpha$ (Type I error) of .05, and a power of .80 , a total of 40 patients (20 per condition) were needed to detect small differences (three points) in catastrophizing thoughts between the groups. We increased the power of this study by including 38 patients per condition.

\section{[FIGURE 1]}

\section{Measures}

\section{Questionnaires}

All patients (control and intervention group) completed written questionnaires prior to the intervention (baseline), upon completion of the intervention (4 weeks), and at 3-month follow-up. Since the intervention mainly focused on opposing dysfunctional complaint-related cognitions and behaviors (see also Table 1), these were our primary outcome measures. Abdominal pain was expected to be influenced indirectly through changes in these cognitions and behaviors. In the baseline questionnaire, the patients were asked to indicate whether or not they ever suffer from any of the following gastrointestinal (GI) symptoms: constipation, diarrhea, bloating, flatulence, and abdominal rumbling. At baseline, 4-week, and 3-month follow-up, the following measures were administered.

\section{[TABLE 1.]}

\section{Cognitive Scale for Functional Bowel Disorders}

The Cognitive Scale for Functional Bowel Disorders (CSFBD) is an illness-specific scale that measures GI symptom-related dysfunctional cognitions commonly experienced by IBS patients [29]. The scale items were derived from Automatic Thought Diaries of IBS patients and then categorized into the themes: anxiety, control, pain, perfectionism, anger/frustration, self-efficacy, social approval, embarrassment/shame, heightened sensitivity to social rules and norms, and self-nurturance. Psychometric analysis produced a 25 -item scale with high reliability and validity and minimal social desirability contamination. No steady scale components were found for the Dutch version [29]. The total score ranges from 31 to 217, with higher scores reflecting more dysfunctional thoughts.

\section{Irritable Bowel Syndrome Quality of Life Questionnaire}

The Irritable Bowel Syndrome Quality of Life Questionnaire (IBS-QoL) is a validated disease-specific instrument that measures the impact of IBS on various aspects of life [30]. It includes questions about food avoidance, social functioning, work functioning, emotional health, mental health, sleep, energy, physical 
Oerlemans, S., Cranenburgh, O. van, Herremans, P.J., Spreeuwenberg, P., Dulmen, S. van. Intervening on cognitions and behavior in irritable bowel syndrome: a feasibility trial using PDAs. Journal of Psychosomatic Research: 2011, 70(3), 267-277

roles, and sexual relations. The IBS-QoL consists of 34 items graded on a five-point Likert-type scale. The items are divided into eight subscales: Dysphoria, Interference with activity, Body image, Health worry, Food avoidance, Social, Sexual, and Relationships. The total score ranges from 0 to 100, with lower scores reflecting better quality of life. We recoded the scores, so higher scores reflect better quality of life on each subscale, which is in line with the direction of CSFBD scores (i.e., the higher the score, the more prevalent the concept under study, being either CSFBD-assessed dysfunctional thoughts or IBS-QoL-assessed quality of life).

\section{The Pain Catastrophizing Scale}

The Pain Catastrophizing Scale (PCS) measures the degree of pain catastrophizing thoughts. It consists of 13, five-point Likert-scaled, items and is mostly used as a total score. Sullivan [31] proposed three dimensions of pain catastrophizing: feelings of helplessness when in pain, rumination about pain symptoms, and magnification of pain-related complaints. On each dimension, subscale scores can be calculated. Higher scores denote a higher degree of catastrophizing. In previous studies, psychometric properties of the PCS appeared adequate [32], [33], [34] and [35]. The PCS has been shown to have good reliability and validity in a clinical population [32].

\section{Abdominal pain}

Abdominal pain was measured using a one-item scale measuring pain severity derived from a previous study in IBS [36]. Patients indicated their current abdominal pain on a five-point Likert-type scale ranging from 1 (no pain) to 5 (serious interfering pain).

\section{Electronic diary}

Complementary to the questionnaires, intervention group patients maintained electronic diaries. The diaries employed in this study were programmed into a PDA (Qtek 2020, Windows Mobile 2003 with flash). Diary questions were presented on a touch screen (see Fig. 2 for an example). Items were constructed according to the Experience Sampling Method [37]. Patients completed three diaries daily. The morning diary assessed quality of sleep, current severity of complaints, and intended activities for the day. An afternoon (alarm-controlled) diary assessed the current severity of complaints and inquired about the patients' accomplished activities, cognitions, and feelings. The evening diary assessed again the current severity of complaints as well as satisfaction with activity level and achievements of that day. Most items were answered on a Likert-type scale, while other questions required answering by brief open responses to be typed on a small keyboard presented on the screen of the PDA. The number of items in the morning, afternoon (alarm-controlled), and evening diary was 9, 15, and 9, respectively.

\section{[FIGURE. 2.]}

\section{Procedure}

GPs were asked to participate by screening their patients based on the IBS ICPC code. The GPs confirmed the diagnoses and checked whether all patients had any comorbidity. They were asked to inform the eligible patients and, if the patient wanted to participate, to notify the researcher, i.e., a psychologist with experience in treating somatic patients in a cognitive-behavioral way. Participants were also recruited via an announcement on the website of the Dutch IBS Association. Interested patients received a brochure and a screening questionnaire consisting of the ROME-III criteria, demographic characteristics, and comorbidity (either medical, psychological, or both). Patients' answers were evaluated independently by two psychologists. When they agreed that a particular patient fulfilled the ROME-III criteria, did not suffer from any comorbidity, and did not receive any other psychological (pharmaceutical) treatment, the patient was included in the study. After receiving informed consent, patients were allocated at random to either the intervention or the control group. All patients received standard care from their GP and completed questionnaires at three time points. Following the Dutch guideline in general practice, this standard care consisted of reassurance, dietary advice, and education [38]. All patients were compensated for their participation with a small gift voucher. 
Oerlemans, S., Cranenburgh, O. van, Herremans, P.J., Spreeuwenberg, P., Dulmen, S. van. Intervening on cognitions and behavior in irritable bowel syndrome: a feasibility trial using PDAs. Journal of Psychosomatic Research: 2011, 70(3), 267-277

Complementary to the three written questionnaires, intervention group patients completed diaries three times daily on a PDA for 4 weeks. During a personal instruction meeting at the patient's house, the PDA was handed out and the procedure and purpose of the study were explained. Every day patients completed three diaries, one in the morning immediately after waking, and one in the evening right before going to sleep. The third, afternoon (alarm-controlled) diary, was assessed randomly between 1 and 3 p.m. after a short message service (SMS) signal. It took patients about 2 min to complete a diary. After the patient had completed the diary, the data were saved on the server and were immediately accessible to the psychologist. Subsequently, during Weeks 2-4, patients received situational feedback based on CBT from a psychologist. This feedback was sent to the patients via SMS. The feedback was provided by one of three junior researchers/psychologists (SO, OvC, $\mathrm{PJH})$ under the supervision of a senior researcher/psychologist (SvD). All feedback was saved and analyzed afterwards.

The transfer of the data between the server and PDA and vice versa was encrypted. In case the computer got stolen, data could not be looked into, since they were not saved on the PDA itself. Data captured from the PDA were transferred to a PC where software imported data into SPSS. Standby assistance during the study to handle (technical) problems was available.

\section{Intervention}

Patients with IBS were randomized to receive either standard care only (control group) or standard care and an additional cognitive-behavioral intervention (intervention group). Intervention group patients received situational feedback on their diaries from a psychologist during 3 weeks. The afternoon diaries served to generate input relevant for formulating feedback. A strict feedback protocol by which specific answers on specific diary questions from the patients generated specific kinds of feedback made it easy to apply and replicate the feedback. The feedback was focused on IBS complaints, catastrophizing thoughts, dysfunctional cognitions, and avoidance behavior, and was mainly based on cognitive-behavior therapy. A protocol to standardize the feedback was developed based on existing literature (e.g., Refs. [19] and [39]) including an informative book on IBS for patients, written by a consortium of Dutch IBS experts [40]. For pragmatic reasons, the protocol was divided into five sets of topics which were structured in a hierarchical way, with the first set of topics (cognitions, emotions, and activities) as the most important to intervene on. If, given the input of the patient, the first-order set of topics was not applicable, feedback was provided on the second-order set of topics (eating and drinking) and so on. The purpose of the feedback was to (1) stimulate self-management, (2) provide reassurance and comfort, and (3) give general information about (coping with) IBS. Table 1 shows the protocol with topics of intervention goals and examples of feedback.

\section{Objectives}

In the current study, we tested the hypothesis that IBS patients receiving the e-health intervention would improve (1) dysfunctional cognitions, (2) quality of life, and (3) complaint severity more than standard care alone.

\section{Statistical analysis}

Analyses were conducted in MLwiN 2.02 to determine whether the standard GP care combined with the CBT intervention on PDAs was more effective than standard GP care alone. Data were analyzed using multilevel analysis [41] with (the level of) abdominal pain severity, dysfunctional cognitions, quality of life, and catastrophizing thoughts as outcome measures and the group condition, time, and sociodemographic variables as independent, predictor variables. Analysis with repeated measurements was performed using multilevel analysis, a modern version of the repeated measures ANOVA, which does not require every measurement in every participant at every time point to be available. Multilevel does take into account that different (repeated) measures are clustered within patients. All estimated means were corrected for autocorrelation between measurements within individuals and standardized for patient's gender, education, age, and diarrhea. All differences between means were tested with the Wald test. 
Oerlemans, S., Cranenburgh, O. van, Herremans, P.J., Spreeuwenberg, P., Dulmen, S. van. Intervening on cognitions and behavior in irritable bowel syndrome: a feasibility trial using PDAs. Journal of Psychosomatic Research: 2011, 70(3), 267-277

\section{RESULTS}

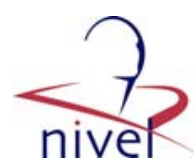

\section{Sample characteristics}

Of the 76 included patients, 38 in the control group and 37 in the intervention group, completed the initial questionnaire. In the control group, 36 patients completed the second measurement and 35 completed all measurements. In the intervention group, 36 patients completed the second measurement and 26 patients completed all measurements. A flowchart is shown in Fig. 1. Sociodemographic characteristics of the total sample are shown in Table 2. At baseline, control group patients reported significantly more diarrhea problems than intervention group patients. Because of this group difference, apart from sociodemographics, diarrhea was also controlled for throughout the analyses. Changes over time within both groups are shown in Table 3. As all 37 intervention group patients completed the intervention and completed diaries three times a day for 4 weeks, the e-health intervention seems feasible.

\section{[TABLE 2]}

\section{Effects of PDA intervention at 4-week (short-term) and 3-month (long-term) follow-up}

\section{Dysfunctional cognitions}

No significant short- or long-term effects on dysfunctional cognitions were found between the intervention and control group (last two columns of Table 3).

\section{IBS Quality of life}

A between-group comparison of the difference scores between baseline and 4 weeks confirmed that there was significantly more overall quality-of-life improvement in the intervention group $(\chi 2=4.08, \mathrm{P}<.05$, $\mathrm{df}=1$ ). At long-term, no significant differences between group effects were found on IBS quality of life.

\section{Catastrophizing thoughts}

A between-group comparison of the difference scores between baseline and 4 weeks confirmed that there was significantly more improvement in catastrophizing thoughts $(\chi 2=9.33, \mathrm{P}<.01, \mathrm{df}=1)$. This improvement in the intervention group persisted at 3-month follow-up $(\chi 2=7.06, \mathrm{P}<.01, \mathrm{df}=1)$.

\section{Abdominal pain}

There was significantly more pain improvement in the intervention group than in the intervention group between baseline and 4-week follow-up $(\chi 2=5.44, \mathrm{P}<.05, \mathrm{df}=1)$. No significant long-term between-group effects were found.

\section{DISCUSSION}

\section{Key findings}

The aim of the present RCT was to establish the feasibility and the efficacy of a cognitive-behavioral ehealth intervention on PDAs on the self-management of IBS patients.

This study has demonstrated that IBS patients receiving the e-health intervention improved in the shortterm their catastrophizing thoughts, quality of life, and abdominal pain more than standard care alone. Improvements in catastrophizing thoughts persisted at 3-month follow-up.

\section{[TABLE 3]}

\section{Possible mechanisms and explanation}

How CBT influences GI symptoms like IBS can be explained in different ways. Drossman's biopsychosocial model of IBS, for example, integrates early life factors (e.g., genetic predisposition and environmental influences) with later psychosocial factors, physiological functioning, and their interaction via the CNS/ENS axis [42]. This global model proposes that the combined and integrated effects of altered physiology and psychosocial functioning affect how GI symptoms are experienced, the individual's response to symptoms, and, ultimately, the outcome (e.g., taking medications, physician visits, daily 
Oerlemans, S., Cranenburgh, O. van, Herremans, P.J., Spreeuwenberg, P., Dulmen, S. van. Intervening on cognitions and behavior in irritable bowel syndrome: a feasibility trial using PDAs. Journal of Psychosomatic Research: 2011, 70(3), 267-277

functioning, or quality of life). The cognitive-behavioral model of IBS postulates that GI symptoms and symptom-related distress are perpetuated by an interaction among psychological (i.e., cognitions, emotions, and behavior), social (i.e., support, modeling), and physiological factors [43]. The model emphasizes that the manner in which an individual psychologically responds to recurrent GI symptoms (i.e., serious disease attributions, hypervigilance) and general life events in turn affects emotional response (e.g., anxiety, fear, embarrassment, guilt, depression), gut motility and visceral hypersensitivity, GI symptoms, and behavioral coping.

In the present study, patients were allocated to the treatment groups randomly, which is generally the best way to control for the various types of possible bias and confounding factors that might lead to falsepositive treatment results [44] and [45]. However, randomization theoretically also bears the possibility that inequalities between groups might occur by chance [46]. Indeed, we found that diarrhea was significantly more often reported in the control group than in the intervention group. As, potentially, improvements could be caused by differences between the study groups, we controlled for diarrhea in all analyses. None of the sociodemographic characteristics differed between the two groups. Additionally, the between-group comparison of the baseline symptom measures yielded only one difference, i.e., intervention group patients suffered more from rumination. Therefore, because of this single difference, we are confident that both patient groups belong to the same patient population.

The importance of the concept of quality of life for IBS has been emphasized. It therefore has been proposed that quantification of quality of life should be an integral part of the outcome measures in treatment trials [47]. The Rome II committees [48] and regulatory agencies [49] recommend that subjective global end points (e.g., patients thoughts, beliefs, and feelings about IBS) should be considered as primary outcome variables. The follow-up measurement in our study showed that the improvement in abdominal pain returned to baseline measures. However, within-group improvements in constipation, quality of life, dysphoria, dysfunctional thoughts about IBS, catastrophizing thoughts, and helplessness persisted at longterm. Between-group comparisons revealed robust long-term effects in catastrophizing thoughts, while the short-term effects in IBS quality of life and abdominal pain had disappeared at long-term. This is consistent with the findings of Drossman et al. [47], where patients often report that "the pain is still there but I'm managing it better." Physicians and mental health professionals recognize this to be a clinically meaningful response [47].

Although the intervention is based on general cognitive-behavioral principles that have been shown to be effective in treating IBS, an important factor to consider is the translation of these techniques into short feedback messages. This may not have resulted in adequate learning and self-implementation and therefore only provides improvements in abdominal pain on a short-term period. Further refinement of the techniques, advice on implementation of the cognitive-behavioral principles, and perhaps a follow-up on the feedback may help patients better apply this self-management method resulting in improvements of this intervention for a longer period.

It has been proposed that a supportive and empathic physician-patient relationship by itself can be associated with the alleviation of patients' symptoms [16] and [50]. The active component(s) of the intervention may be the patient's expectation of improvement, attention from a health care provider, or encouragement to resume healthy functioning. Alternatively, it is possible that this intervention relieves feelings of helplessness and dysfunctional thoughts, which may promote a reduction in physical discomfort. For future studies using PDAs, a second control group is recommended to determine whether the improvements in psychological measures can be attributed to the CBT, to the 'buddy function' of the PDA, or to completing daily diaries.

\section{Comparison to other published studies}

Several studies have been carried out to determine the effects of CBT in IBS. Our findings are in line with those of Van Dulmen et al. [19] who compared group cognitive therapy with a waiting-list control group. A total of 27 patients with IBS were allocated to receive group therapy. Patients who attended the cognitive group therapy showed significantly more improvement in their abdominal complaint scores than did those in the control group. However, there were no differences in psychological well-being. Drossman et al. [47] assessed the clinical efficacy of CBT compared to education in female patients with moderate to severe functional bowel disorders. Participants received either CBT or education for 12 weeks. They found that CBT is significantly more effective than education and stated that CBT is effective in treating women with 
Oerlemans, S., Cranenburgh, O. van, Herremans, P.J., Spreeuwenberg, P., Dulmen, S. van. Intervening on cognitions and behavior in irritable bowel syndrome: a feasibility trial using PDAs. Journal of Psychosomatic Research: 2011, 70(3), 267-277

functional bowel syndrome, including IBS. Our results are also consistent with psychological treatments for chronic pain that often result in improved quality of life and reduced pain-related disability [51]. Nevertheless, some studies show contradictory findings in comparison with our findings. In a review of seven different controlled trials of CBT with IBS sufferers, mixed results were found [52]. Four trials showed that CBT relieved bowel symptoms more than did standard medical care [53] or a waiting list [19], [54] and [55]. Three other studies found no difference between CBT and a control condition [56], [57] and [58].

\section{Feasibility}

Although we did not have 3-month follow-up data from every patient in the intervention group, all 37 intervention group patients did complete the intervention and completed diaries three times a day for 4 weeks and completed the 4-week follow-up. Given this, the e-health intervention itself, i.e., completing diaries three times a day for 4 weeks, seems feasible. However, the higher dropout rate at 3-month followup in the intervention group might also indicate a high burden of completing questionnaires as well as diaries for 4 weeks which control group patients did not experience. Future studies should assess this possibility more carefully. Besides, given the high drop-out rate at follow-up, our analysis cannot be understood as an intention-to-treat analysis. Post hoc comparisons on baseline and follow-up measurements between dropouts and completers indicate that, overall, dropouts experienced a more favorable IBS-related quality of life, which could be attributed completely to more positive scores on the subscale measuring the quality of relationships. Unfortunately, we do not know why patients dropped out, but we can rule out the fact that the dropouts were the worse cases.

\section{Strengths and limitations}

The present study has a number of strengths. Patients were randomly assigned to either the control or the intervention group. In addition, two global outcome measures specifically developed to capture IBS were used in this study, i.e., IBS-QoL and CSFBD. Moreover, we increased the power of this study by including almost 40 patients per condition. The trial benefited from a fairly high rate of follow-up, with $94.7 \%$ completing the post-intervention measurement and 80.3\% completing the 3-month follow-up.

Several limitations of the study need to be considered. First, included patients represented subgroups of IBS, e.g., diarrhea or constipation-prone IBS. Results for specific subgroups might differ. Therefore it is difficult to generalize the results. Second, no process measures were included in the study to assess whether participants learn from the provided information/principles or to determine whether they correctly understood and implemented the cognitive and behavioral techniques. However, all information and advice we provided in the feedback were listed and analyzed. The feedback protocol was strongly linked to specific answers on specific questions in the electronic diary, so the feedback can be applied and replicated easily. Third, we did not confine the study to patients who had been resistant to treatment, as in the study by Heymann-Monnikes et al. [50]. Such patients may be very different from the patients seen in routine gastroenterological clinics. It is known that CBT is most appropriate for those patients who are considerably distressed by their symptoms, are open to the idea that psychological factors play some role in their difficulties, and are willing to participate in this approach [17]. And, finally, it is possible that the patients we recruited from the Dutch IBS Association had no official IBS diagnosis. Not every patient was diagnosed by their GP. However, all patients did fulfil the ROME-III criteria.

\section{Future research and conclusion}

Future research should focus on a longer intervention period and maybe a replication of the provided feedback. The inclusion of process measures could be very useful to determine whether patients actually learn something new and whether they understand and are able to implement the learned information. Furthermore, long-term follow-up assessments are critical in determining the efficacy of any intervention. If treated patients return to their former levels of functioning within a year of treatment, the value of these interventions is limited.

In conclusion, our data indicate that a cognitive-behavioral intervention on pocket-type computers is feasible and efficacious for improving abdominal pain, quality of life, and catastrophizing thoughts in patients with IBS. The only sustainable improvement was on catastrophizing thoughts, which was also the basis of this trial. However, the other improvements waned over time, so this intervention may therefore need regular follow-ups to sustain the desired outcome. The finding that the long-term change in 
Oerlemans, S., Cranenburgh, O. van, Herremans, P.J., Spreeuwenberg, P., Dulmen, S. van. Intervening on cognitions and behavior in irritable bowel syndrome: a feasibility trial using PDAs. Journal of Psychosomatic Research: 2011, 70(3), 267-277

catastrophizing thoughts was not mirrored in other symptoms may suggest that the improvement in

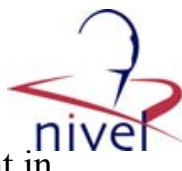
thoughts is not directly relevant to symptom improvement. Follow-up research should unravel underlying mechanisms to find out what (combination of) elements of the intervention (i.e., symptom monitoring, CBT, e-diary, or minimal contact therapy) can be held responsible for the positive results.

\section{Acknowledgments}

The authors wish to thank the participating patients and GPs as well as the Dutch IBS patient organization for their contribution to this project.

\section{REFERENCES}

[1] WG Thompson, GF Longstreth, DA Drossman, KW Heaton, EJ Irvine and SA Muller-Lissner, Functional bowel disorders and functional abdominal pain, Gut 45 (1999), pp. 43-47.

[2] CJ Bijkerk, NJ De Wit, WAB Stalman, JA Knottnerus, A Hoes and JWM Muris, Irritable bowel syndrome in primary care: The patients' and doctors' views on symptoms, aetiology and management, Can J Gastroenterol 17 (2003), pp. 363-368.

[3] WG Thompson, KW Heaton, GT Smyth and C Smyth, Irritable bowel syndrome in general practice: Prevalence, characteristics, and referral, Gut 46 (2000), pp. 78-82.

[4] J.W. Muris, A. Oberndorff-Klein Wolthuis, F. Pace, L. Rodrigo, R. Stockbrügger and M.H. Vatn, Epidemiological aspects of irritable bowel syndrome in Europe and North America, Digestion 64 (2001), pp. 200-204.

[5] JW Muris, R Starmans, GH Fijten, HF Crebolder, TF Krebber and JA Knottnerus, Abdominal pain in general practice, Fam Pract 10 (1993), pp. 387-390.

[6] A Foxx-Orenstein, IBS Review and what's new, Med Gen Med 8 (2006), p. 20.

[7] GF Longstreth, WG Thompson, WD Chey, LA Houghton, F Mearin and RC Spiller, Functional bowel disorders, Gastroenterol 130 (2006), pp. 1480-1491.

[8] GF Longstreth and DA Drossman, New developments in the diagnosis and treatment of irritable bowel syndrome, Curr Gastroenterol Rep 4 (2002), pp. 427-434.

[9] J Jailwala, TH Imperiale and K Kroenke, Pharmacologic treatment of the irritable bowel syndrome: a systematic review of randomised, controlled trials, Ann Int Med 133 (2000), pp. 136-147.

[10] BD Cash and WD Chey, Advances in the management of irritable bowel syndrome, Curr Gastroenterol Rep 5 (2003), pp. 468-475.

[11] JA Mains and FR Scogin, The effectiveness of self-administered treatments: A practice-friendly review of the research, Psychother Pract 59 (2003), pp. 237-246.

[12] RW Marrs, A meta-analysis of bibliotherapy studies, Am J Comm Psychol 23 (1995), pp. 843-871.

[13] RA Gould and GA Clum, A meta-analysis of self-help treatment approaches, Clin Psychol Rev 13 (1993), pp. 169-186.

[14] AM Van Dulmen, JFM Fennis, HGA Mokkink and G Bleijenberg, The relationship between complaintrelated cognitions in referred patients with irritable bowel syndrome and subsequent health care seeking behaviour in primary care, Fam Pract 13 (1996), pp. 12-17.

[15] MM Heitkemper, ME Jarrett, RL Levy, KC Cain, RL Burr and A Feld et al., Self-management for women with irritable bowel syndrome, Clin Gastroenterol Hepatol 2 (2004), pp. 585-596.

[16] American College of Gastroenterology Task Force on IBS, An evidence-based systematic review in the management of irritable bowel syndrome, Am J Gastroenterol 104 (suppl 1) (2009).

[17] J Hutton, Cognitive behaviour therapy for irritable bowel syndrome. Review in depth, Eur J Gastroenterol Hepatol 17 (2005), pp. 11-14.

[18] B Greene and EB Blanchard, Cognitive therapy for irritable bowel syndrome, J Consult Clin Psychol 62 (1994), pp. 576-582.

[19] AM Van Dulmen, JFM Fennis and G Bleijenberg, Cognitive-behavioural group therapy for irritable bowel syndrome: Effects and long-term follow-up, Psychosom Med 58 (1996), pp. 501-514.

[20] PM Boyce, NJ Talley, B Balaam, NA Koloski and G Truman, A randomized controlled trial of cognitive behavior therapy, relaxation training, and routine clinical care for the irritable bowel syndrome, Am J Gastroenterol 98 (2003), pp. 2209-2218.

[21] JM Lackner, BM Quigley and EB Blanchard, Depression and abdominal pain in IBS patients: The mediating role of catastrophizing, Psychosom Med 66 (2004), pp. 435-441.

[22] ML Peters, MJ Sorbi, DA Kruise, JJ Kerssens, PF Verhaak and JM Bensing, Electronic diary assessment of pain, disability and psychological adaptation in patients differing in duration of pain, Pain 84 (2000), pp. 181-192. 
Oerlemans, S., Cranenburgh, O. van, Herremans, P.J., Spreeuwenberg, P., Dulmen, S. van. Intervening on cognitions and behavior in irritable bowel syndrome: a feasibility trial using PDAs. Journal of Psychosomatic Research: 2011, 70(3), 267-277

[23] DA Kruise, MJ Sorbi, JM Bensing, AM van Dulmen and PMM Spreeuwenberg, Electronic guidance of behavior relevant to migraine: A pilot in low-back pain. In: EGM Couturier and MJ Sorbi, Editors, Headache and Migraine, Anglo Dutch Migraine Association, Utrecht (2004).

[24] A Payne and EB Blanchard, A controlled comparison of cognitive therapy and self-help support groups in the treatment of irritable bowel syndrome, J Consult Clin Psychol 63 (1995), pp. 779-786.

[25] M Jarrett, M Heitkemper, KC Cain, M Tuftin, EA Walker and EF Bond et al., The relationship between psychological distress and gastrointestinal symptoms in women with irritable bowel syndrome, Nurs Res 47 (1998), pp. 154-161.

[26] M Csikszentmihalyi and R Larson, Validity and reliability of the Experience-Sampling Method, J Nerv Mental Dis 175 (1987), pp. 526-536.

[27] AA Stone and S Shiffman, Ecological Momentary Assessment (EMA) in behavioral medicine, Ann Behav Med 16 (1994), pp. 199-202.

[28] M Morren, S van Dulmen, J Ouwerkerk and J Bensing, Compliance with momentary pain measurement using electronic diaries: A systematic review, Eur J Pain 13 (2009), pp. 354-365.

[29] BB Toner, N Stuckless, A Ali, F Downie, S Emmott and D Akman, The development of a cognitive scale for functional bowel disorders, Psychosom Med 60 (1998), pp. 492-497.

[30] DL Patrick, DA Drossman, IO Frederick, J Dicesare and KL Puder, Quality of life in persons with irritable bowel syndrome (development and validation of a new measure), Dig Dis Sci 43 (1998), pp. 400411. Full Text via CrossRef | View Record in Scopus | Cited By in Scopus (168)

[31] MJ Sullivan, The Pain Catastrophizing Scale: development and validation, Psychol Assess 7 (1995), pp. 524-532.

[32] G Crombez, C Eccleston, F Baeyens and P Eelen, When somatic information threatens, catastrophizing thinking enhances attentional interference, Pain 75 (1998), pp. 187-198.

[33] G Crombez, JW Vlaeyen, PH Heuts and R Lysens, Pain-related fear is more disabling than pain itself: Evidence on the role of pain related fear in chronic back pain disability, Pain 80 (1999), pp. 329-339.

[34] S Van Damme, G Crombez, P Bijttebier, L Goubert and B Van Houdenhove, A confirmatory factor analysis of the Pain Catastrophizing Scale: Invariant factor structure across clinical and non-clinical populations, Pain 96 (2002), pp. 319-324.

[35] JWS Vlaeyen, SM Geurts, AMJ Kole-Snijders, JA Schuerman, NH Groenman and H Eek van, What do chronic pain patients think of their pain? Towards a pain cognition questionnaire, Br J Clin Psychol 28 (1990), pp. 383-394.

[36] AM Van Dulmen, JFM Fennis, HGA Mokkink, HGM Van der Velden and G Bleijenberg, Doctors' perception of patients' cognitions and complaints in irritable bowel syndrome at an out-patient clinic, J Psychosom Res 38 (1994), pp. 581-590.

[37] In: PAEG Delespaul, Editor, Assessing Schizophrenia in Daily Life, Universitaire Pers Maastricht, Maastricht (1995).

[38] HE Van der Horst, JS Meijer, JWM Muris, B Sprij, FMPB Visser and ACM Romeijnders et al., NHGStandaard Prikkelbare darm syndroom, [GP Guideline irritable bowel syndrome], Huisarts Wet 44 (2001), pp. 58-65.

[39] HE Van der Horst, FG Schellevis, JT Van Eijk and G Bleijenberg, Managing patients with irritable bowel syndrome in general practice. How to promote and reinforce self-care activities, Patient Educ Couns 35 (1998), pp. 149-156.

[40] In: S Kollaard and H Vinke, Editors, Handboek Prikkelbare Darm Syndroom, [Care book Irritable bowel syndrome] (2006).

[41] AH Leyland and PP Groenewegen, Multilevel modelling and public health policy, Scand J Public Health 31 (2003), pp. 267-274. Full Text via CrossRef | View Record in Scopus | Cited By in Scopus (50)

[42] DA Drossman, WE Whitehead and M Camilleri, Irritable bowel syndrome: A technical review for practice and guideline development, Gastroenterology 112 (1997), pp. 2120-2137.

[43] BB Toner, ZV Segal, S Emmott, D Myran, A Ali and I DiGasbarro et al., Cognitive-behavioral group therapy for patients with irritable bowel syndrome, Int J Group Psychother 48 (1998), pp. 215-243.

[44] $\mathrm{CH}$ Hennekens and SH Buhring, Analysis of epidemiologic studies: Evaluating the role of bias. In: $\mathrm{CH}$ Hennekens and SH Buhring, Editors, Epidemiology in Medicine, Little, Brown and Company, Boston (Mass) (1987), pp. 272-286.

[45] $\mathrm{CH}$ Hennekens and SH Buhring, Analysis of epidemiologic studies: Evaluating the role of confounding. In: $\mathrm{CH}$ Hennekens and SH Buhring, Editors, Epidemiology in Medicine, Little, Brown and Company, Boston (Mass) (1987), pp. 287-323.

[46] SJ Pocock, Methods of randomization, Clinical Trials. A Practical Approach, John Wiley and Sons, Chichester (1983), pp. 66-89. 
Oerlemans, S., Cranenburgh, O. van, Herremans, P.J., Spreeuwenberg, P., Dulmen, S. van. Intervening on cognitions and behavior in irritable bowel syndrome: a feasibility trial using PDAs. Journal of Psychosomatic Research: 2011, 70(3), 267-277

[47] DA Drossman, BB Toner, WE Whiteheas, NE Diamant, CB Dalton and S Duncan et al., Cognitivebehavioral therapy versus education and desipramine versus placebo for moderate to severe functional bowel disorders, Gastroenterology 125 (2003), pp. 19-31.

[48] SJO Veldhuyzen van Zanten, NJ Talley, P Bytzer, K Klein, PJ Whorwell and AR Zinsmeister, Design of treatment trials for the functional gastrointestinal disorders. In: DA Drossman, E Corazziari, NJ Talley, WG Thompson and WE Whitehead, Editors, Rome II: Functional Gastrointestinal Disorders: Diagnosis, Pathophysiology, and Treatment (2nd ed), Degnon Associates, Inc, McLean (Va.) (2000), pp. 577-622.

[49] WE Whitehead, E Corrazziari, R Prizont, JR Senior, WG Thompson and SJO Veldhuyzen van Zanten, Definition of a responder in clinical trials for functional gastrointestinal disorders. In: DA Drossman, E Corazziari, NJ Talley, WG Thompson and WE Whitehead, Editors, Rome II: Functional Gastrointestinal Disorders: Diagnosis, Pathophysiology, and Treatment (2nd ed.), Degnon Associates, Inc., McLean (Va.) (2000), pp. 623-627.

[50] I Heymann-Monnikes, R Arnold, I Florin, C Herda, S Melfsen and H Mönnikes, The combination of medical treatment plus multicomponent behavioural therapy is superior to medical treatment alone in the therapy of irritable bowel syndrome, Am J Gastroenterol 95 (2000), pp. 981-994.

[51] A Gamsa, The role of psychological factors in chronic pain: 1: A half century of study, Pain 57 (1994), pp. 5-15.

[52] LA Allen, JI Escobar, PM Lehrer, MA Gara and RL Woolfolk, Psychosocial treatments for multiple unexplained physical symptoms: A review of the literature, Psychosom Med 64 (2002), pp. 939-950. Full Text via CrossRef | View Record in Scopus | Cited By in Scopus (63)

[53] G Shaw, ED Srivastava, M Sadlier, P Swann, JY James and J Rhodes, Stress management for irritable bowel syndrome: A controlled trial, Digest 50 (1991), pp. 36-42.

[54] PM Lynch and E Zamble, A controlled behavioral treatment study of irritable bowel syndrome, Behav Ther 20 (1989), pp. 509-523.

[55] DF Neff and EB Blanchard, A multi-component treatment for irritable bowel syndrome, Behav Ther 18 (1987), pp. 70-83.

[56] P Bennett and S Wilkinson, A comparison of psychological and medical treatment of the irritable bowel syndrome, Br J Clin Psychol 24 (1985), pp. 215-216.

[57] EB Blanchard, SP Schwarz, JM Suls, MA Gerardi, L Scharff and B Greene et al., Two controlled evaluations of multi-component psychological treatment of irritable bowel syndrome (study 1 ), Behav Res Ther 30 (1992), pp. 175-189.

[58] EB Blanchard, SP Schwarz, JM Suls, MA Gerardi, L Scharff and B Greene et al., Two controlled evaluations of multi-component psychological treatment of irritable bowel syndrome (study 2), Behav Res Ther 30 (1992), pp. 175-189. 
Oerlemans, S., Cranenburgh, O. van, Herremans, P.J., Spreeuwenberg, P., Dulmen, S. van. Intervening on cognitions and behavior in irritable bowel syndrome: a feasibility trial using PDAs. Journal of Psychosomatic Research: 2011, 70(3), 267-277

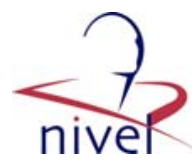

\section{FIGURES AND TABLES}

Fig. 1. Flowchart.

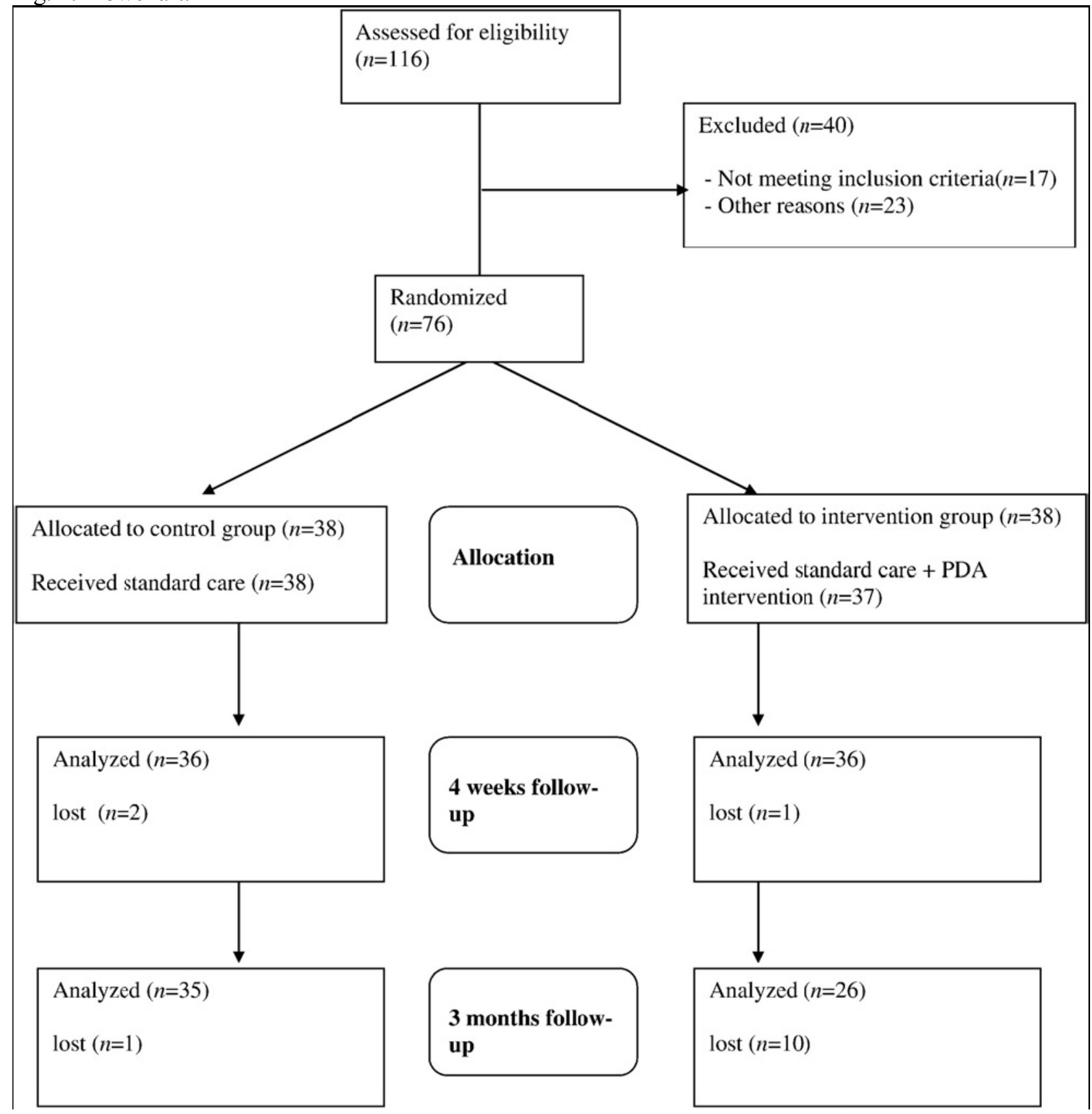


Table 1

Hierarchical feedback protocol in five sets of topics with specific goals and examples

\begin{tabular}{|c|c|c|}
\hline Topics & Goals & Examples \\
\hline $\begin{array}{l}\text { 1. Cognitions, emotions, } \\
\text { and activities }\end{array}$ & $\begin{array}{l}\text { - Avoid unnecessary worries } \\
\text { Explain that worry and anxiety about complaints that are in themselves harmless can sustain the } \\
\text { complaints. A thorough physical examination revealing no serious underlying organic cause } \\
\text { for the complaints should be considered as the first step in breaking through the vicious circle. } \\
\text { - Do not let the complaints determine your life } \\
\text { Explain that sometimes people unnecessarily avoid or refrain from all kinds of activities because } \\
\text { of their complaints. This so-called avoidance behavior can aggravate the complaints by causing } \\
\text { stress and a vicious circle develops. There is no need for people with IBS to avoid any activities for } \\
\text { medical reasons. } \\
\text { - Get enough physical exercise } \\
\text { Stress the importance of physical exercise. Too little exercise can lead to all kinds of health } \\
\text { problems, such as abdominal complaints like constipation. }\end{array}$ & $\begin{array}{l}\text { - Examine your anxiety. Try to formulate the source of this anxiety as } \\
\text { explicitly as possible. } \\
\text { - Think about the activities you avoid or refrain from as a consequence } \\
\text { of the complaints, consider whether you want to avoid or refrain from each } \\
\text { particular activity. } \\
\text { - Make arrangements (if necessary, together with other people) to start a } \\
\text { certain activity, specify what and when. } \\
\text { - Take some kind of physical exercise each day, e.g., half an hour walking } \\
\text { or cycling. } \\
\text { - Use the stairs instead of an elevator or use your bicycle instead of your } \\
\text { car or public transport. }\end{array}$ \\
\hline 2. Eating and drinking & $\begin{array}{l}\text { - General education } \\
\text { Explain how abdominal complaints can be influenced by irregular eating habits, e.g., skipping } \\
\text { a meal, eating irregularly. } \\
\text { - Food and complaints } \\
\text { Explain that in some patients abdominal complaints can be diet related. This is especially the case } \\
\text { with complaints such as diarrhea and constipation. Keeping a diary gives the patient information on } \\
\text { which type of food or drink aggravates the complaints and, subsequently, whether elimination } \\
\text { of the offending food or drink reduces the complaints. }\end{array}$ & $\begin{array}{l}\text { - Do not skip a meal. } \\
\text { - Take daily meals at approximately the same time. } \\
\text { - Drink enough, at least } 1.51 \text { of liquid a day. }\end{array}$ \\
\hline 3. Relaxation & $\begin{array}{l}\text { - Reduce stress } \\
\text { Explain how stress can aggravate complaints and that people are not always aware of experiencing } \\
\text { stress when, in fact, it is occurring (e.g., by moving house, starting a new job, etc.). If stress } \\
\text { evokes or aggravates physical complaints people tend to pay more attention to the complaints than } \\
\text { to the stress itself. A vicious circle can be the result, as is the case with anxiety. }\end{array}$ & $\begin{array}{l}\text { - Consider whether the increasing complaints could be a sign of some strain } \\
\text { you are experiencing. } \\
\text { - Concentrate your efforts on the stress and not on the complaints. } \\
\text { - Try to find out what has caused the stress, what can be done to reduce the } \\
\text { stress, and who can help you to cope with the stress. }\end{array}$ \\
\hline 4. Secondary complaints & $\begin{array}{l}\text { - Reduce secondary complaints } \\
\text { Explain that many people with irritable bowel syndrome are tired. Possible causes of this are pain, } \\
\text { bad sleeping behavior, and stress. }\end{array}$ & $\begin{array}{l}\text { - If you are tired, you are more sensitive to pain. Go to bed at regular times } \\
\text { and try to sleep } 8 \mathrm{~h} \text { a night. }\end{array}$ \\
\hline $\begin{array}{l}\text { 5. Abdominal } \\
\text { complaints }\end{array}$ & $\begin{array}{l}\text { - Reduce abdominal complaints and provide reassurance } \\
\text { Inform the patient that the origin of the complaints is not yet known and explain that it is not likely } \\
\text { that an organic explanation will be found in due time. Many factors can contribute to the complaints, } \\
\text { e.g., a history of gastrointestinal infection, stress, life events, dietary problems, too little physical } \\
\text { exercise, anxiety about the complaints, etc. }\end{array}$ & $\begin{array}{l}\text { - Try to identify factors that might influence your abdominal complaints and } \\
\text { learn how to cope with these factors and the complaints themselves. }\end{array}$ \\
\hline
\end{tabular}


Oerlemans, S., Cranenburgh, O. van, Herremans, P.J., Spreeuwenberg, P., Dulmen, S. van. Intervening on cognitions and behavior in irritable bowel syndrome: a feasibility trial using PDAs. Journal of Psychosomatic Research: 2011, 70(3), 267-277

Fig. 2. Screenshot of diary (in Dutch).

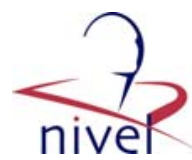

\section{(6) Opties (A) (X) \\ Toen $\mathrm{k}$ vanmorgen wakker werd, had k last van...}

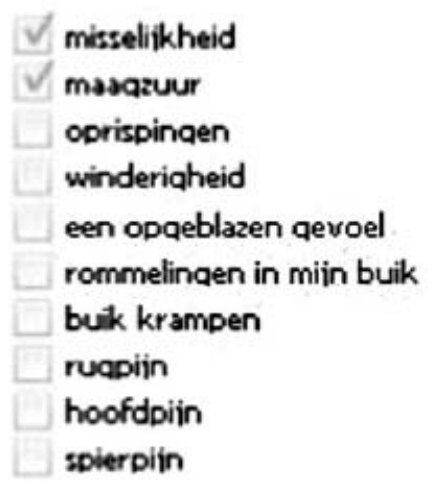

\section{data saved}

Table 2

Sample characteristics by group

\begin{tabular}{llll}
\hline Variable & $\begin{array}{l}\text { Control group } \\
(n=39)\end{array}$ & $\begin{array}{l}\text { Intervention group } \\
(n=37)\end{array}$ & $P$ \\
\hline Age in years (S.D.) & $40.6(15.5)$ & $35.9(11.7)$ & .15 \\
Gender (\% female) & $76.9 \%$ & $91.9 \%$ & .14 \\
Education in years & & & .88 \\
Low & $12.8 \%$ & $13.5 \%$ & \\
Middle & $51.3 \%$ & $54.1 \%$ & .28 \\
High & $35.9 \%$ & $29.7 \%$ & \\
Marital status & & & \\
Not married/cohabiting & $28.2 \%$ & $43.2 \%$ & \\
Married/cohabiting & $64.1 \%$ & $54.1 \%$ & .19 \\
Divorced & $7.7 \%$ & $2.7 \%$ & .02 \\
Gastrointestinal & & & .16 \\
$\quad$ symptoms & & $43.2 \%$ & .08 \\
Constipation & $25.6 \%$ & $18.9 \%$ & .91 \\
Diarrhea & $46.2 \%$ & $81.1 \%$ & \\
Bloating & $64.1 \%$ & $67.6 \%$ & \\
Flatulence & $87.2 \%$ & $73.0 \%$ & \\
Rumbling & $69.2 \%$ & & \\
\hline
\end{tabular}


Table 3

Mean baseline (T0), 4-week (T1), and 3-month follow-up (T2) scores on primary and secondary outcome measures for intervention group (T0: $n=37$; T1: $n=36$; T2: $n=26$ ) and control group (T0: $n=39 ; \mathrm{T} 1: n=36 ; \mathrm{T} 3: n=35$ )

\begin{tabular}{|c|c|c|c|c|c|c|c|c|c|}
\hline Outcome measure & Group & T0 & $95 \% \mathrm{CI}$ & $\mathrm{T} 1$ & $95 \% \mathrm{CI}$ & $\mathrm{T} 2$ & $95 \% \mathrm{CI}$ & $\mathrm{T} 0-\mathrm{T} 1$ & T0-T2 \\
\hline \multirow[t]{2}{*}{ CSFBD Total } & Intervention & 79.82 & $61.39-98.26$ & $70.83^{*}$ & $52.16-89.49$ & $68.74^{1+1+}$ & $49.13-88.34$ & 8.99 & 11.08 \\
\hline & Control & 77.74 & $60.57-94.90$ & 75.90 & $59.21-92.59$ & 72.08 & $54.39-89.77$ & 1.84 & 5.66 \\
\hline \multirow[t]{2}{*}{ IBS-QoL Total } & Intervention & 74.76 & $65.56-83.95$ & $78.60^{*}$ & $69.29-87.91$ & $78.46^{+}$ & $69.08-87.85$ & $-3.84^{\bar{i}}$ & -3.70 \\
\hline & Control & 80.53 & $72.31-88.76$ & 79.62 & $72.02-87.22$ & 80.21 & $72.01-88.41$ & 0.91 & 0.32 \\
\hline \multirow[t]{2}{*}{ PCS Total } & Intervention & 26.93 & $21.36-32.50$ & $23.57^{* *}$ & $18.44-28.70$ & $22.21^{++}$ & $16.83-27.59$ & $3.36^{i i j}$ & $4.72^{i i i}$ \\
\hline & Control & 21.99 & $16.71-27.26$ & 24.13 & $19.03-29.23$ & 23.10 & $18.13-28.07$ & -2.14 & -1.11 \\
\hline \multirow[t]{2}{*}{ Abdominal pain } & Intervention & 1.98 & $1.57-2.38$ & $1.46^{* * * *}$ & $1.03-1.90$ & 1.75 & $1.31-2.19$ & $0.52^{j i}$ & 0.23 \\
\hline & Control & 1.99 & $1.51-2.47$ & 1.89 & $1.44-2.33$ & 2.03 & $1.55-2.50$ & 0.1 & -0.04 \\
\hline
\end{tabular}

All estimated means are corrected for autocorrelation between measurements within individuals and standardized for patient's gender, education, age, and diarrhea.

Within-group differences: T0 vs. T1: ${ }^{*} P<.05, \chi^{2} \geq 3.84 ;{ }^{* *} P<01, \chi^{2} \geq 6.64 ; *^{* *} P<001, \chi^{2} \geq 10.83$.

Within group differences: T0 vs. T2: ${ }^{+} P<.05, \chi^{2} \geq 3.84 ;{ }^{+1} P<01, \chi^{2} \geq 6.64 ;{ }^{+}{ }^{+1} P<.001, \chi^{2} \geq 10.83$.

T0-T1 indicates the short-term effect; T0-T2, the long term effect. \# refers to $P$ value of tests on between-group differences, " $P<.05, " i i \bar{P}<.01$.

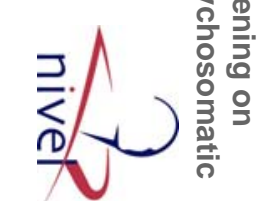

